\title{
Variations in Canadian Rates of Hospitalization for Ambulatory Care Sensitive Conditions
}

Maria Sanchez, Smitha Vellanky, Jeremy Herring, Jun Liang and Hui Jia

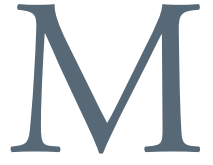

any chronic conditions, such as diabetes or asthma, can be successfully managed in the community. Appropriate screening, ongoing monitoring, prescribing of medications, providing patient education and other supportive measures that help keep these conditions under control are provided in primary healthcare settings. However, sometimes people with such conditions require hospitalization. Although not all admissions for these conditions are avoidable, timely and effective ambulatory care can potentially reduce the risk of hospitalization by possibly preventing or controlling the onset of an illness or condition or by managing the chronic condition (World Health Organization 2005). These conditions are often referred to as ambulatory care sensitive conditions (ACSC).

The conditions used to define ACSC in this analysis include angina, asthma, chronic obstructive pulmonary disorder (COPD), diabetes, grand mal status and other epileptic convulsions, heart failure and pulmonary edema and hypertension (Canadian Institute for Health Information 2008). This is based on an adaptation of the widely used definition of ACSC by Billings et al. (1993).

Research shows that variations in ACSC hospitalization rates may be related to factors such as differences in access to and quality of primary healthcare (Ansari et al. 2006; Caminal et al. 2004). They may also be due to differences in community- or hospital-based practice patterns or other factors.

\section{ACSC Hospitalization Rates Are Falling}

In 2006-2007, ACSC hospitalizations accounted for one out of every eight (13\%) medical hospitalizations (excluding surgical, obstetrical, newborn and mental health cases) for patients younger than 75 years of age in Canada, outside Quebec. The highest proportion of these hospitalizations was for COPD (Figure 1). However, over the past six years, the number of people admitted to hospital for an ACSC has decreased. After adjusting for population growth and aging, the hospitalization rate for ACSC dropped by 22\% between 2001-2002 and 2006-2007. The analysis shows that, with the exception of COPD (which increased by $1 \%$ ), hospital admissions for each of the other six ACSC decreased during the six-year period. The largest drop (almost 50\%) observed was for hospitalizations for angina. In 2006-2007, 351 people out of every 100,000 were admitted to a hospital for an ACSC.

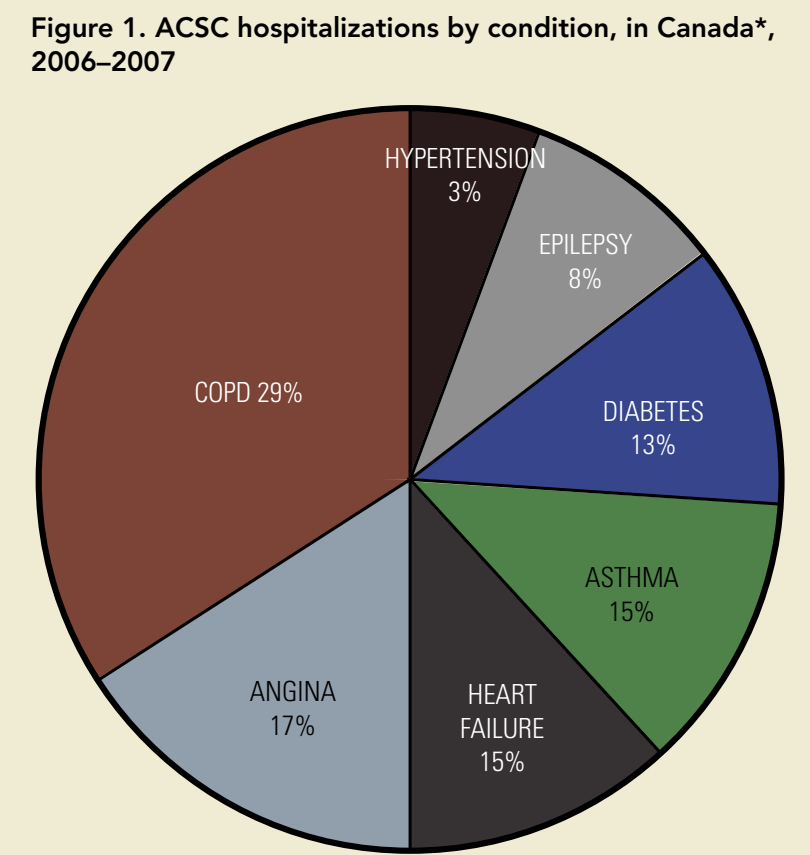

This graph shows the distribution of ACSC hospitalizations by condition. Of the estimated 87,500 hospitalizations, COPD and angina accounted for nearly half of the total. *Data from Quebec were not available for 2006-2007 at the time of publication.

Source: Discharge Abstract Database, Canadian Institute for Health Information.

\section{Geographical Variations in ACSC Hospitalization Rates}

Although the overall ACSC age-adjusted hospitalization rate decreased over the six-year period, the rates varied markedly across the country. In 2006-2007, hospitalization rates in 
Ontario and British Columbia were lower than the overall average, while rates in all other provinces and territories, outside Quebec, were higher. At the regional level, a five-fold difference in rates was observed (Figure 2).

Sizable variations in ACSC hospitalization rates between rural and urban areas were also observed. In 2006-2007, the overall age-adjusted hospitalization rate for all ACSC in rural areas, outside Quebec, was about 510 per 100,000, 60\% higher than in urban areas, where the rate was 318 per 100,000 population. Hospitalization rates for each of the seven chronic conditions were also higher in rural areas. For example, hospitalization rates for hypertension were significantly higher in rural areas compared with urban areas: 22 versus seven per 100,000 population, respectively.

\section{Variations by Income}

Certain patient and system characteristics can affect hospitalization rates. A number of studies have identified an inverse relationship between neighbourhood income and ACSC hospitalizations (Agha et al. 2007; Billings et al. 1993; Booth and Hux 2004; Roos et al. 2005). Neighbourhood income is frequently used as a proxy measure of individuals' income levels (Borugian et al. 2005). To help quantify neighbourhood income, households are often divided into quintiles according to their gross income. Each quintile represents $20 \%$, or one fifth, of all households. In 2006-2007, outside Quebec, hospitalization rates decreased as income levels rose (Figure 3). In the lowest income group, the age-adjusted rate for all ACSC hospitalizations was 521 per 100,000 , the highest among all income quintiles. This rate was more than twice as high as the rate in the most affluent income group, which had a rate of 234 per 100,000 population.

\section{Conclusions}

A number of different factors are related to the observed variations in ACSC hospitalization rates across the country. Factors associated with these rates include, but are not limited to, age, gender, income level and geographic location. Yet the reasons underlying these variations are not well understood. However, as ACSC hospitalizations are potentially preventable, research suggests that issues related to access and well-functioning primary healthcare services may play an important role in the management of these conditions (Ansari et al. 2006; Caminal et al. 2004). Initiatives designed to enhance access to and the

Figure 2. Regional variations in ACSC hospitalization rates, in Canada*, 2006-2007

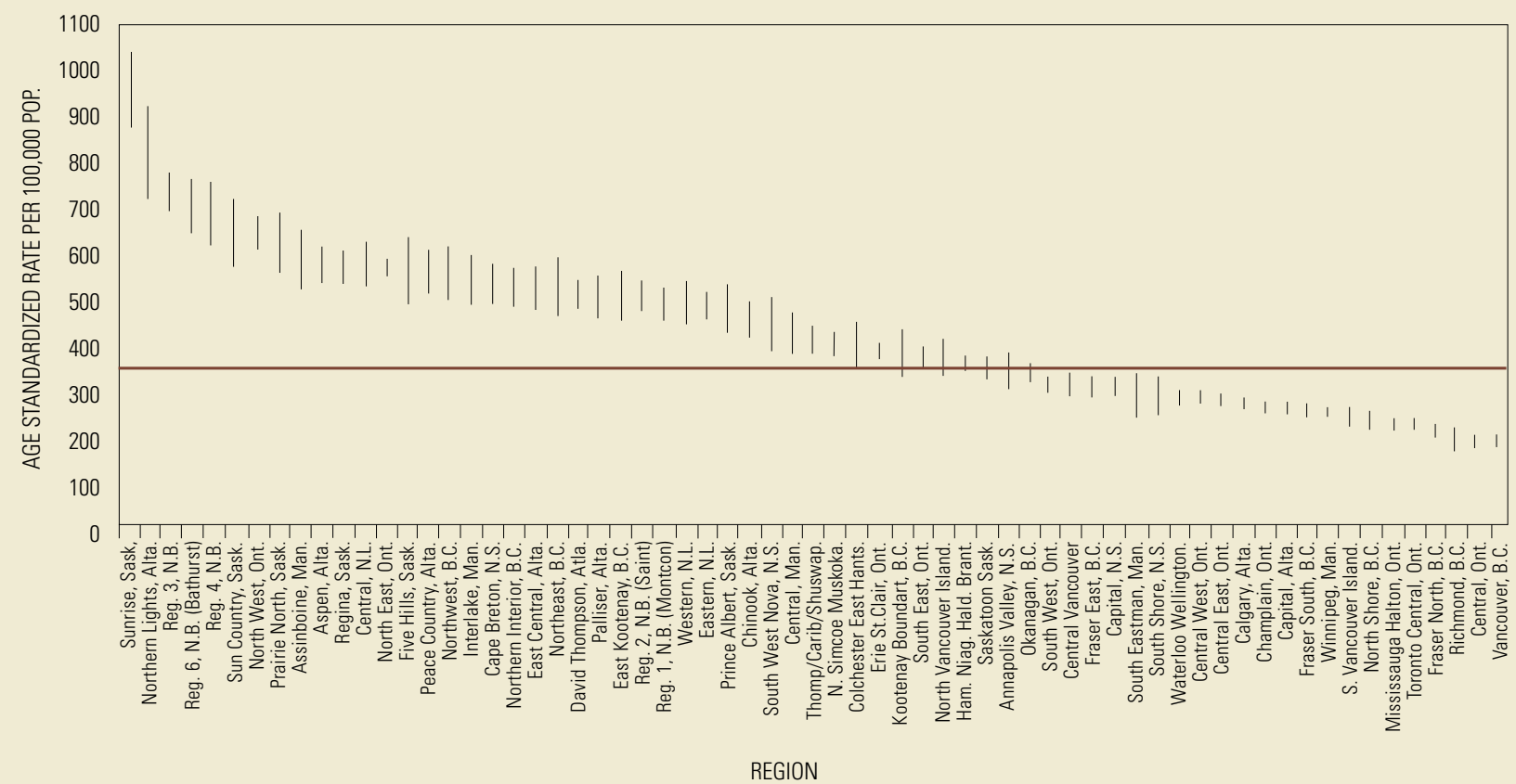

This graph shows the age-standardized hospitalization rates for ACSC among Canadians, outside Quebec, younger than 75 years of age in 2006 -2007 based on where patients lived, not where they were treated. The solid line indicates the overall ACSC hospitalization rate (351 per 100,000 population). *Regions not shown were excluded due to small numbers. Data from Quebec were not available for 2006-2007 at the time of publication

Source: Discharge Abstract Database, Canadian Institute for Health Information. 
Figure 3. Overall ACSC hospitalizations by income quintiles*, in Canada ${ }^{\dagger}$, 2006-2007

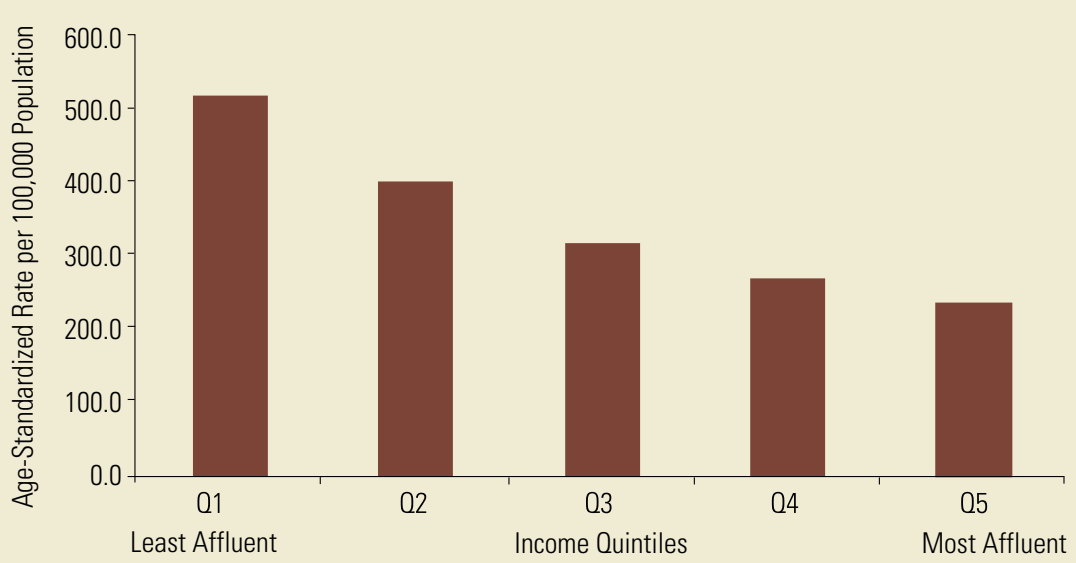

This graph shows the age-standardized rates for overall ACSC hospitalizations by income quintile among Canadians younger than 75 years of age, outside Quebec, for 2006-2007. Hospitalization rates decreased across income quintiles.

${ }^{\dagger} 01$

using the 2001 Canadian census.

* Data from Quebec were not available for 2006-2007 at the time of publication.

Source: Discharge Abstract Database, Canadian Institute for Health Information
Sensitive Conditions." European Journal of Public Health 14: 246-51.

Campbell, S., D. Reeves, E. Kontopantelis, E. Middleton, B. Sibbald and M. Roland. 2007. "Quality of Primary Care in England with the Introduction of Pay for Performance." New England Journal of Medicine 357(2): 181-90.

Canadian Institute for Health Information. 2008. Health Indicators 2008. Ottawa: Author.

Cyr, M.-C., A.C. Martens, D. Berbiche, S. Perreault and L. Blais. 2006. "Continuity of Care in the Ambulatory Treatment of Adolescents with Asthma." Journal of Adolescent Health 39(6): 926. e11-17.

Roos, L.L., R. Walld, J. Uhanova and R. Bond. 2005. "Physician Visits, Hospitalizations, and Socioeconomic Status: Ambulatory Care Sensitive Conditions in a Canadian Setting." Health Services Research 40(4): $1167-$ 85.

Sargious, P. 2007. Primary Health Care Transition Fund, Chronic Disease Prevention and Management: Glass Half Empty, Glass Half Full. Ottawa: Health Canada. Retrieved March 18, 2008.

quality of primary healthcare services have been implemented in various countries, including Canada (Campbell et al. 2007; Cyr et al. 2006; Sargious 2007; Starfield et al. 2005). These initiatives include improving access to services, educating patients, implementing electronic health records, promoting coordination and continuity of care, setting standards for treatment of major chronic diseases and improving models of primary healthcare delivery. HQ

\section{References}

Agha, M.M., R.H. Glazier and A. Guttmann. 2007. "Relationship between Social Inequalities and Ambulatory Care Sensitive Hospitalizations Persists for Up to 9 Years among Children Born in a Major Canadian Urban Center." Ambulatory Pediatrics 7(3): 258-62.

Ansari, Z. J.N. Laditka and S.B. Laditka. 2006. "Access to Health Care and Hospitalization for Ambulatory Care Sensitive Conditions." Medical Care Research and Review 63(6): 719-41.

Billings, J., L. Zeitel, J. Lukomnik, T.S. Carey, A.E. Blank and L. Newman. 1993. "Datawatch: Impact of Socioeconomic Status on Hospital Use in New York City." Health Affairs 12(1): 162-73.

Booth, B.L. and J.E. Hux. 2003. "Relationship between Avoidable Hospitalizations for Diabetes Mellitus and Income Level." Archives of Internal Medicine 163(1): 101-06.

Borugian, M.J., J.J. Spinelli, G. Mezel and R. Wilkins. 2005. "Childhood Leukemia and Socioeconomic Status in Canada." Epidemiology 16(4): 526-31.

Caminal, J., B. Starfield, E. Sánchez, C. Casanova and M. Morales. 2004. "The Role of Primary Care in Preventing Ambulatory Care <http://www.healthcanada.gc.ca/>.

Starfield, B., L. Shi and J. Macinko. 2005. "Contribution of Primary Care to Health Systems and Health.” Milbank Quarterly 83(3): 457502.

World Health Organization. 2005. The Impact of Chronic Disease in Canada. Geneva: Author. Retrieved March 17, 2008. <http://www. who.int $>$.

\section{About the Authors}

Maria Sanchez, BSc, MSc, is an analyst in Health Reports, providing lead methodological and analytical support to the Health Indicators report as well as other special reports.

Smitha Vellanky, BSc, MSc, is an analyst in Health Reports, providing analytical and methodological support to the Health Indicators report as well as other special reports.

Jeremy Herring, BSc, MSc, is a senior analyst in Health Indicators, providing lead methodological and analytical support to Health Indicators report as well as other special reports.

Jun Liang, BSc, MSc is an analyst in Health Indicators, providing analytical support to Health Indicators report as well as other special reports.

Hui Jia, BSc, MSc, is an analyst in Health Reports, providing analytical and methodological support to the Health Indicators report as well as other special reports. 